\title{
Sequences of islet amyloid polypeptide precursors of an old world monkey, the pig-tailed macaque (Macaca nemestrina), and the dog (Canis familiaris)
}

\author{
S. Ohagi ${ }^{1}$, M. Nishi ${ }^{1}$, G. I. Bell ${ }^{1,2}$, J.W. Ensinck ${ }^{3}$ and D. F.Steiner ${ }^{1,2}$ \\ ${ }^{1}$ Department of Biochemistry and Molecular Biology, and ${ }^{2}$ Howard Hughes Medical Institute, The University of Chicago, Chicago \\ ${ }^{3}$ Department of Medicine, University of Washington, Seattle, USA
}

\begin{abstract}
Summary. The 37-amino acid islet amyloid polypeptide represents the major protein component present in islet amyloid deposits. Although the presence of islet amyloid is a characteristic pathological feature of the islets of humans, monkeys and cats with Type 2 (non-insulin-dependent) diabetes mellitus, it is not found in the islets of diabetic rats, mice or dogs. To further explore the molecular basis for these species differences in amyloid deposition we have used a polymerase chain reaction based method to clone cDNAs encoding the monkey (Macaca nemestrina) and dog (Canis familiaris) islet amyloid polypeptide precursors. The predicted amino acid sequence of the monkey precursor is $96 \%$ identical to that of the human protein; differences include one replacement in the signal peptide and three in the islet amyloid polypeptide domain. The sequence of the dog precursor is most closely re-
\end{abstract}

lated to that of the cat protein ( $85 \%$ identity); the sequences of dog and cat islet amyloid polypeptide differ only at two positions and are identical in the region of amino acids $20-29$, the region thought to be primarily responsible for amyloidogenesis. Thus, amino acid residues in addition to those at positions 20-29 may facilitate the aggregation of islet amyloid polypeptide. The presence of amyloid deposits in some dog pancreatic endocrine tumours suggests that the dog protein can be amyloidogenic, perhaps due to elevated expression of islet amyloid polypeptide by the tumours relative to normal islets.

Key words: Islet amyloid polypeptide precursors, monkey (Macaca nemestrina), dog (Canis familiaris), amyloidogenic properties.
Islet amyloid polypeptide (IAPP) is a normal secretory product of the pancreatic Beta cell and is a major constituent of amyloid deposits in patients with Type 2 (non-insulin-dependent) diabetes mellitus [1-4]. The levels of IAPP in the islet and in the peripheral circulation are about 1\% those of insulin [5]. The presence of amyloid deposits is the most common pathological lesion seen in the islets of human subjects with Type 2 diabetes. These deposits are also present in the islets of aged individuals although the degree of amyloidosis is much less than that seen in diabetic subjects. Although IAPP is predicted to have hormone-like properties, its normal physiological function(s) are unknown. However, its pathological deposition in the islets disrupts the normal architecture of the islet and may account at least in part for the impaired Beta-cell function seen in patients with Type 2 diabetes. Amyloid deposits have also been noted in the islets of diabetic monkeys [6,7] and cats [8]; the protein component of cat islet amyloid has been isolated and partially sequenced and shown to be IAPP [3]. Amyloid deposits also occur in the islets of the diabetic degu, a South American rodent related to the guinea pig; however, the protein component of degu islet amyloid is insulin rather than IAPP [9].

Although IAPP-derived amyloid deposits are a feature of diabetes mellitus in humans, monkeys and cats, amyloidosis is not present in the islets of diabetic mice, rats or dogs. The molecular basis for this difference in islet pathology may be related to the structure of the IAPP molecule, especially amino acid sequence differences in the putative fibril-forming region (residues 20-29) [10]. As part of our ongoing studies of the biosynthesis and functional properties of IAPP as well as its role in the impairment of islet function, we have determined the sequences of the IAPP precursors in two useful animal models of diabetes, one of which is characterized by the presence of islet amyloid deposits, the old world monkey, Macaca nemestrina, and the other which is not, the dog, Canis familiaris.

\section{Materials and methods}

Genomic DNA was prepared from peripheral blood lymphocytes obtained from a single monkey and from two dogs. RNA was isolated from the pancreases of a fasted monkey and dog using the gua- 
A. Pig-tailed Macaque Islet Amyloid Polypeptide

GAGGGGGTAAATATTCCAGTGGATACAAGCTTGGGCTCTTTTCTTGAAGCTTTCTTTCTGTCAGAGGCATTTGCTGATATTGCTGACGTTGAAACAGTAAAAGAAAATTTGAGAAGCA

-22 -20 Signal peptide -10 1 NH ${ }^{-t e r m i n a l ~ p r o p e p t i d e ~}$

Met Cys Ile Leu Lys Ieu Gln Val phe Leu Ile Val Leu Ser Val Ala Leu Asn His Leu Iys Ala Thr Ero Ile Glu Ser His Gin Val ATG TGC ATT CTG AAG CTG CAA GTA TTT CTC ATC GTG CTC TCT GTT GCA TTA AAC CAT CTG AAA GCT ACA CCC ATT GAA AGT CAT CAG GTG

$10 \quad$ IAPP

20

30

Glu Lys Arg Iys Cys Asn Thr Ala Thr Cys Ala Thr Gln Arg Leu Ala Asn Phe Leu VaI Arg Ser Ser Asn Asn Phe Gly Thr Ile Leu GAA AAG CGG AAA TGC AAC ACT GCC ACG TGT GCA ACG CAG CGC CTG GCA AAT TTT TTA GIT CGT TCC AGC AAC AAC TTT GGT ACC AIT CTC

40 The $50 \quad$ COOH-terminal propeptide

60

67

Ser Ser Thr Asn Val Gly Ser Asp Thr Tyr Gly Lys Arg Asn Ala Val Glu Val Leu Iys Arg Glu Pro Leu Asn Tyr Ieu Pro Leu AM TCA TCT ACC AAC GTG GGA TCC GAT ACA TAT GGC AAG AGG AAT GCA GTA GAG GTT TTA AAG AGA GAG CCA CTG AAT TAC TTG CCC CTT TAG AGGACGATGTAACTCTATAGTTATTGTTTTATGTTCTAGAGATTTCCTGTATAATTTAACACTGCCTTTTTCATTTCCAGTGTGAATATATGGTCTGTGTATCTGATGTTTGTTGCCAGG ACATATACATTGTCAAAAGATTGTTTTATAT

B. Dog Islet Amyloid Polypeptide

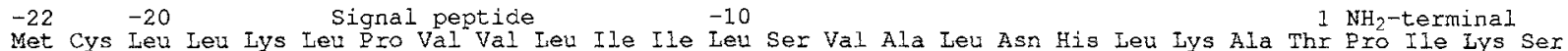
TTGAGAAAA MTG TGT CTC CTG AAG CTG CCA GTA GTT CTC ATC ATA CTC TCG GTT GCA TTA AAC CAT CTG AAG GCT ACT CCC ATT AAA AGT propeptide 10 Gis 10 IAPR propeptide
His Gln Met GIu LYS Arg LYs Cys Asn Thr Ala Thr Cys Ala Thr Gln Arg Leu Ala Asn Phe Leu val Arg Thr Ser Asn Asn Leu Giy
CAC CAG ATG GA AAG CGG AAA TGC AAC ACT GCC ACG TGT GCG ACT CAA CGC CTG GCA AAT TTC TTG GTT CGT ACT AGC AAT AAT CTT GGT 40050 cooH-terminal propeptide 60 Ala Ile Leu Sex Pro Thr Asn Val Gly Ser Asn Thr Tyr Gly Lys Arg Asn Thr Ile Glu Ile Leu Asn Arg Giy PIo Leu Asn Tyr Leu
GCC ATT CTC TCG CCT ACC AAT GTG GGA TCC AAT ACA TAT GGC AAG AGA AAC ACA ATT GAG ATT TrA AAC AGG GGA CCA TTG AAT TAC TTA 67

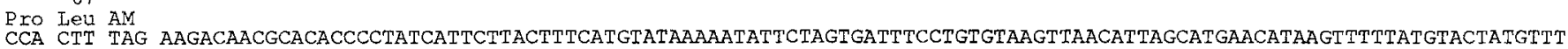

GTTACGACTACATATAAATTGTTATGGTAAGAATTG

Fig.1A,B. Nucleotide and deduced amino acid sequences of monkey (A) and $\operatorname{dog}(\mathbf{B})$ islet amyloid polypeptide (IAPP) precursor cDNAs. The IAPP domain of the precursor is indicated in bold type

nidinium thiocyanate/ $\mathrm{CsCl}$ procedure. Monkey (Macaque nemestrina) tissues were kindly provided by the Regional Primate Research Center at the University of Washington (supported by NIH Grant RR 00166).

\section{Amplification of homologous DNA fragments and rapid amplification of $c D N A$ ends}

Amplification of homologous DNA fragments (AHF) was carried out as described previously [11]. Briefly, a 119-base pair DNA fragment coding for mature IAPP was amplified from genomic DNA by the polymerase chain reaction (PCR) using the following two oligonucleotide primers: 5'-AAGTGCAACACTGCCACATGTGC-3' and 5'-CGCTTGCCATATGTATTGGA-3'. PCR was carried out for 30 cycles as follows: denaturation for $1 \mathrm{~min}$ at $94^{\circ} \mathrm{C}$, annealing for 2 min at $50^{\circ} \mathrm{C}$, and extension for $3 \mathrm{~min}$ at $72^{\circ} \mathrm{C}$. Amplified fragments of the expected size, 119 base pairs, were isolated, subcloned into the HincII site of M13 mp18, and sequenced by the dideoxy nucleotide chain-termination procedure. The remainder of the sequence of the IAPP precursor nucleotide sequence was obtained using the $5^{\prime}$ - and 3'-RACE protocol as described previously [11]. The rapid amplification of cDNA ends (RACE) reaction products were subcloned into the HincII site of pGEM4Z, identified by colony hybridization and sequenced. Sequences of six individual clones were usually determined to rule out any errors due to the amplification procedure.

\section{RNA blotting}

RNA was separated in a $1.5 \%$ agarose- $0.66 \mathrm{~mol} / \mathrm{f}$ formaldehyde gel, blotted onto nitrocellulose filters, and hybridized with nick-translated cDNA. Filters were washed under high stringency conditions $(15 \mathrm{mmol} / 1 \mathrm{l}$ sodium chloride $/ 1.5 \mathrm{mmol} / 1$ sodium citrate $/ 0.1 \%$ sodium dodecyl sulphate, $60^{\circ} \mathrm{C}$ ) and autoradiographed with intensifying screens.

\section{Results}

The sequences of monkey and dog IAPP precursors were deduced from nucleotide sequences generated by a combination of AHF and RACE. The cDNA and predicted amino acid sequences of monkey and $\operatorname{dog}$ IAPP are shown

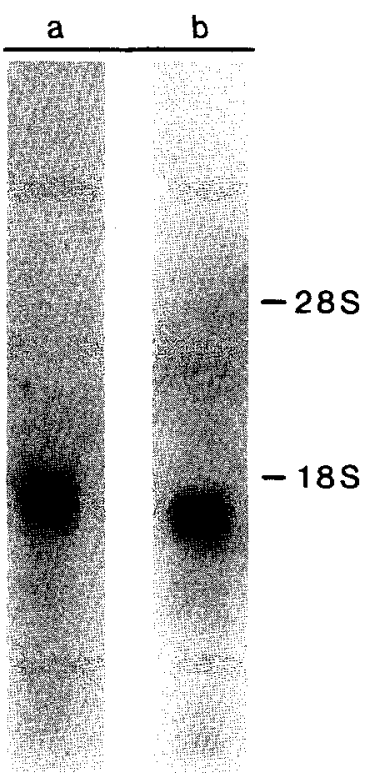

Fig. 2 a,b. RNA blot of monkey and dog islet anyloid polypeptide mRNAs. Five micrograms of poly (A) ${ }^{+}$RNA isolated from monkey (lane a) and dog (lane b) pancreas were electrophoresed, blotted, and hybridized with the corresponding nick-translated cDNA and autoradiographed for $24 \mathrm{~h}$. Positions of $18 \mathrm{~S}$ and $28 \mathrm{~S}$ rRNA are shown 


$$
\begin{array}{lll} 
& \mathrm{NH}_{2} \text {-terminal } & \text { cooH-terminal } \\
\text { Sropeptide } & \text { Islet amyloid polypeptide } & \text { propeptide }
\end{array}
$$

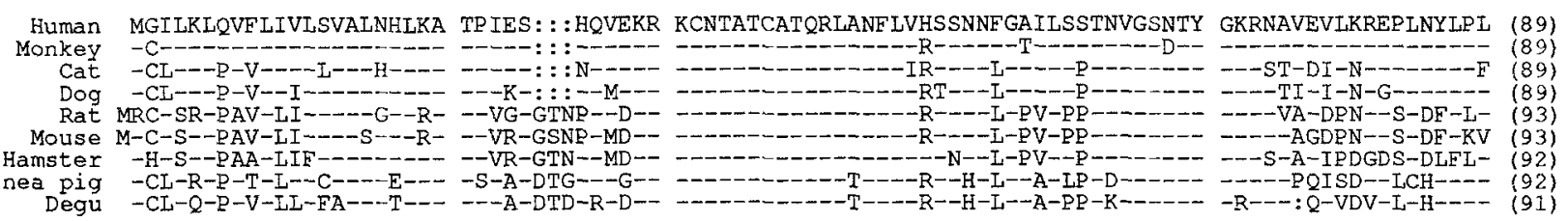

Fig.3. Comparison of deduced amino acid sequences of nine mammalian islet amyloid polypeptide (IAPP) precursors. Signal peptide, IAPP motif and $\mathrm{NH}_{2}$ - and $\mathrm{COOH}$-terminal propeptide segments are indicated. Dashes indicate amino acid residues identical to the

in Figure 1. The sizes of monkey and dog pancreatic IAPP mRNA are 2.2 and 2.1 kilobases, respectively, (Fig. 2) indicating that the nucleotide sequences presented in Figure 1 do not correspond to all of the mRNA.

The amino acid sequences of IAPP precursors of nine mammals are shown in Figure 3. The percent identity and similarity between these sequences are summarized in Table 1 . The identity varies from $59-96 \%$. The sequence of the IAPP portion of the precursor is more highly conserved than the other domains which is consistent with IAPP being the only biologically active peptide derived from this protein.

\section{Discussion}

Islet amyloid deposits in association with diabetes have been reported in several species of monkeys, including Macaca nemestrina [6,7]. In longitudinal studies of $\mathrm{Maca}$ ca nigra, it was noted that islet amyloidosis preceded development of overt diabetes and the extent of amyloid de- human sequence. Colons indicate gaps inserted to generate this alignment. The length of each precursor is shown. The sequences of the IAPP precursors are from references $11,15-17$

position correlated well with the degree of metabolic deterioration [12]. Furthermore, in view of the relatively close relationship of monkeys to humans, the macaque provides a highly suitable animal model for human Type 2 diabetes. The sequence of the monkey IAPP precursor is very similar to that of the human $(96 \%$ identity and similarity). Remarkably, no differences were found in the $\mathrm{NH}_{2}$ - and $\mathrm{COOH}$-terminal propeptide regions between the monkey and human precursors. In the critical fibrilforming region, i. e. residues 20-29, monkey IAPP differs by only one amino acid from that of human: residue 25 , which is alanine in human and cat IAPP, is changed to threonine. Since the substitution of proline for alanine at position 25 in human IAPP had only minor effects on its in vitro fibril-forming ability [10], the threonine substitution at this position in monkey IAPP is unlikely to cause a significant reduction in its amyloidogenic properties.

Although dogs have IAPP immunoreactivity in the Beta cells of their pancreases, diabetic animals do not develop islet amyloidosis. Moreover, a recent report of the partial sequence of dog IAPP indicated that it is very simi-

Table 1. Identity and similarity between mammalian islet amyloid polypeptide (IAPP) peptides and precursors

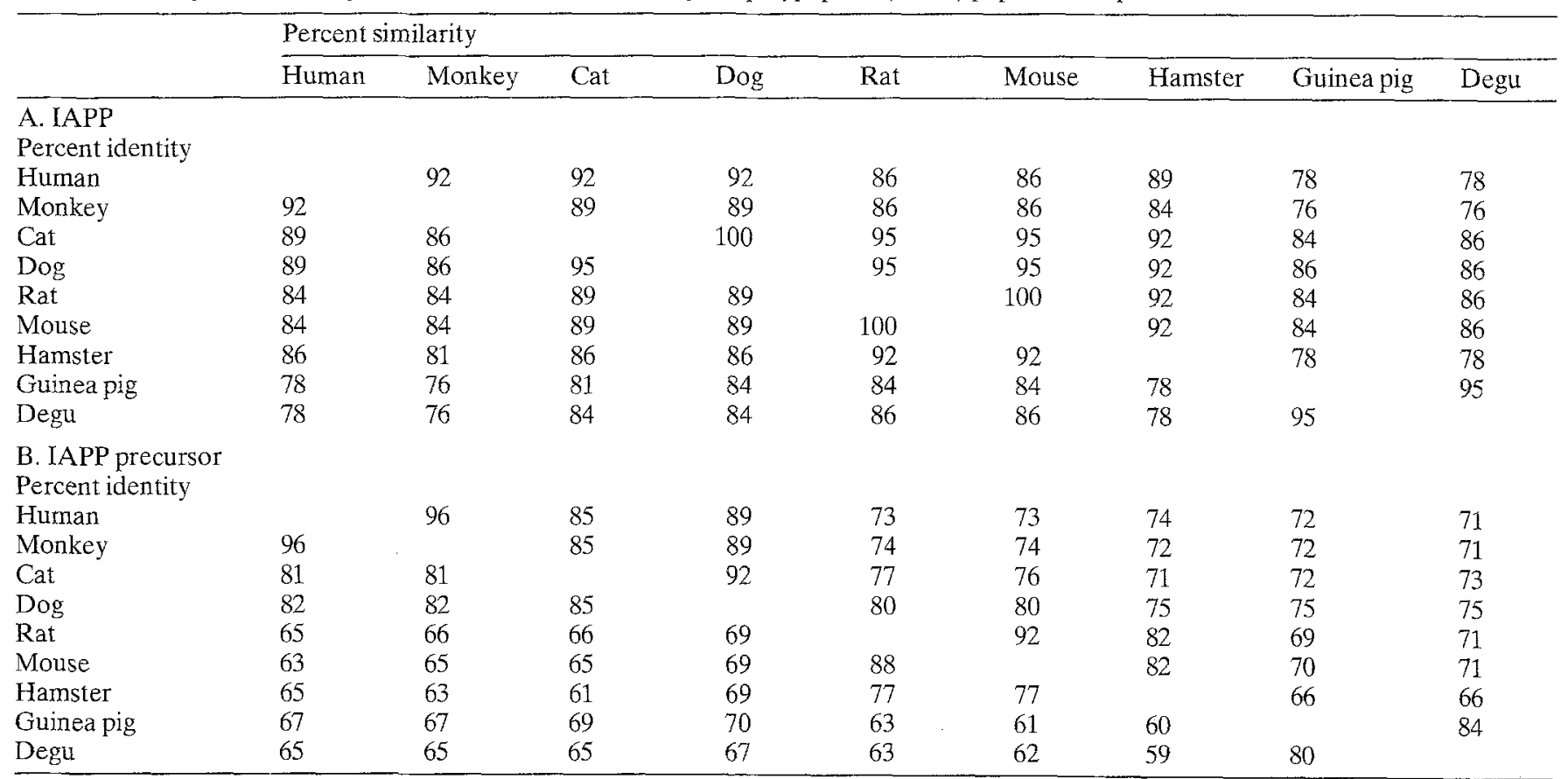

For calculations of percent similarity the following amino acid residues were considered to be equivalent: D, E; F, Y, W; K, R; I, L, M, V; N, Q; and $\mathrm{S}, \mathrm{T}$ 
lar to that of the cat, a species known to have such deposits [13]. Our results confirm that the sequences of dog IAPP and its precursor are closely similar to those of the cat ( $95 \%$ and $85 \%$ identity, respectively [Fig. 3, Table 1]). In the fibril-forming region of IAPP, the amino acid sequence of dog IAPP is identical to that of the cat, as recently reported [13]. However, our sequence differs from that reported by Jordan et al. [13]; there are three nucleotide differences, one of which results in an amino acid difference. Residue 34 of $\operatorname{dog}$ IAPP, which is serine in our sequence and in eight other mammals, is threonine in the report by Jordan et al., perhaps indicating an allelic difference in this species. However, both of the dog samples studied in this work gave identical prepro IAPP sequences. From the results of our present study, the sequence of dog IAPP differs by only two amino acids from that of the cat, at residues 18 and 20. It is possible that one or both of these changes may account for the absence of islet amyloid deposits in diabetic dogs. The mRNA levels in Northern analysis appear to be comparable in dog and monkey pancreases (Fig. 2), but there could be differences in the translational efficiency of these mRNAs in these two species. A recent study demonstrated the presence of IAPP-immunoreactivity in amyloid deposits associated with pancreatic endocrine tumours in dogs [14]. This finding suggests that increased expression of IAPP or its precursor, as may occur in such tumours (18; ref. 19, unpublished results) plays a role in the generation of these amyloid deposits. The reasons for the absence of such deposits in the islets of diabetic dogs remain unclear but, if not the result of reduced levels of expression of IAPP in dogs relative to other species, may indicate that the threshold concentration of IAPP required to initiate the aggregation of dog IAPP into fibrils is higher than that for the cat or human proteins and these levels are not normally exceeded in diabetic dogs whereas they may be in pancreatic endocrine tumours.

Acknowledgements. We thank Mr. P. Gardner for synthesis of oligonucleotides and Ms. F. Rozenfeld for her assistance in preparing this manuscript. Work from this laboratory was supported by NIH Grant DK 13914 and the Howard Hughes Medical Institute.

\section{References}

1. Westermark P, Wernstedt C, Wilander E, Sletten K (1986) A novel peptide in the calcitonin-gene related peptide family as an amyloid fibril protein in the endocrine pancreas. Biochem Biophys Res Commun 140: 827-831

2. Clark A, Lewis CE, Willis AC, Cooper GJS, Morris JR, Reid KBM, Turner RC (1987) Islet amyloid formed from diabetes-associated peptide may be pathogenic in type-2 diabetes. Lancet II: 231-234

3. Westermark P, Wernstedt C, Wilander E, Hayden DW, O'Brien TD, Johnson KH (1987) Amyloid fibrils in human insulinoma and islets of Langerhans of diabetic cat are derived from a neuropeptide-like protein also present in normal islet cells. Proc Natl Acad Sci USA 84: 3881-3885
4. Cooper GJS, Willis AC, Clark A, Turner RC, Sim RB, Reid KBM (1987) Purification and characterization of amyloid-rich pancreases of type 2 diabetic patients. Proc Natl Acad Sci USA 84: 8628-8632

5. Steiner DF, Ohagi S, Nagamatsu S, Bell GI, Nishi M (1991) Perspective: Is islet amyloid polypeptide (amylin) a significant factor in the pathogenesis or pathophysiology of diabetes? Diabetes 40 : 305-309

6. Howard CF Jr (1978) Insular amyloidosis and diabetes mellitus in Macaca nigra. Diabetes 27:357-364

7. Leathers CW, Schedewie HK (1980) Diabetes mellitus in a pigtailed macaque (Macaca nemestrina). J Med Primatol 9; 95-100

8. Yano BL, Hayden DW, Johnson KH (1981) Feline insular amyloid: association with diabetes mellitus. Vet Pathol 18: 621-627

9. Hellman U, Wernstedt C, Westermark P, O'Brien TD, Rathbun WB, Johnson KH (1990) Amino acid sequence from degu islet amyloid-derived insulin shows unique sequence characteristics. Biochem Biophys Res Commun 169: 571-577

10. Westermark P, Engström U, Johnson KH, Westermark GT, Betsholtz C (1990) Islet amyloid polypeptide: Pinpointing amino acid residues linked to amyloid fibril formation. Proc Natl Acad Sci USA 87: 5036-5040

11. Nishi M, Chan SJ, Nagamatsu S, Bell GI, Steiner DF (1989) Conservation of the sequence of islet amyloid polypeptide in five mammals is consistent with its putative role as an islet hormone. Proc Natl Acad Sci USA 86: 5738-5742

12. Howard CF Jr (1986) Longitudinal studies on the development of diabetes in individual Macaca nigra. Diabetologia 29:301-306

13. Jordan K, Murtaugh MP, O'Brien TD, Westermark P, Betsholtz C, Johnson KH (1990) Canine IAPP cDNA sequence provides important clues regarding diabetogenesis and amyloidogenesis in type 2 diabetes. Biochem Biophys Res Commun 169: 502-508

14. O'Brien TD, Westermark P, Johnson KH (1990) Islet amyloid polypeptide and calcitonin gene-related peptide immunoreactivity in amyloid and tumor cells of canine pancreatic endocrine tumors. Vet Pathol 27: 194-198

15. Sanke T, Bell GI, Sample C, Rubenstein AH, Steiner DF (1988) An islet amyloid peptide is derived from an 89-amino acid precursor by proteolytic processing. J Biol Chem 263: 17243-17246

16. Nishi M, Bell GI, Steiner DF (1990) Sequence of a cDNA encading Syrian hamster islet amyloid polypeptide precursor. Nucl Acids Res 18: 6726

17. Nishi M, Steiner DF (1990) Cloning of complementary DNAs encoding islet amyloid polypeptide, insulin, and glucagon precursors from a New World rodent, the degu, Octodon degus. Mol Endocrinol 4: 1192-1198

18. Madsen OD, Nielsen JH, Michelsen B, Westermark P, Betsholtz C, Nishi M, Steiner DF (1991) Islet amyloid polypeptide (IAPP/amylin) and insulin expression are controlled differently in primary and transformed islet cells. Mol Endocrinol 5: 143148

19. Nagamatsu S, Nishi M, Steiner DF (1991) Biosynthesis of islet amyloid polypeptide: Elevated expression in mouse $\beta$ TC 3 cells. $\mathrm{J}$ Biol Chem (in press)

Received: 19 February 1991 and in revised form: 26. April 1991

Dr. D.F.Steiner

Howard Hughes Medical Institute

5841 S. Maryland Ave.

Box 23

Chicago, IL 60637

USA 